\title{
CLINICAL STATUS OF THE AUDITORY STEADY-STATE RESPONSE IN INFANTS
}

De Wet Swanepoel ${ }^{1,2}$

1. Department of Communication Pathology, University of Pretoria, South Africa

2. Callier Center for Communication Disorders, University of Texas at Dallas, USA

\section{Contact details:}

Dept of Communication Pathology

University of Pretoria

South Africa

0002

Tel: +27124202304

Fax: +27 124203517

Email: dewet.swanepoel@up.ac.za 


\section{ABSTRACT}

Pediatric audiology has seen the inclusion of the auditory steady-state response (ASSR) in clinical test-batteries as a valuable diagnostic tool. Its unique stimuli, recording and analysis characteristics allow for applications not previously possible with other auditory evoked responses in infants and young children. Although the longstanding research and clinical validation history of the frequency-specific auditory brainstem response (ABR) make it the current gold standard for estimating hearing thresholds, accumulating evidence is establishing the ASSR as a reliable and accurate tool for the diagnosis of hearing loss in infants. Current test-protocol efficiency and accuracy may be improved by including the ASSR to supplement ABR data and to cross-check test results. This article reviews the ASSR and its current clinical applications and limitations for determining hearing thresholds in infants and young children.

\section{LEARNING OBJECTIVES}

1. The learner will be able to identify the clinical strengths and limitations of the ASSR in infants

2. The learner will be able to list the complementary roles of the ASSR in the recommended ABR test-battery for infants

\section{KEYWORDS}

Auditory steady-state response, objective audiometry, auditory brainstem response, infant hearing loss, pediatric audiology, auditory evoked potentials 


\section{ABBREVIATIONS}

ABR - Auditory Brainstem Response

AEP - Auditory Evoked Potential

AM - Amplitude Modulation

ASSR - Auditory Steady-State Response

EEG - Electroencephalographic

FFT - Fast Fourier Transform

FM - Frequency-Modulation

OAE - Oto-Acoustic Emission

UNHS - Universal Newborn Hear Screening

\section{INTRODUCTION}

Auditory evoked potentials (AEP) have served as important tools in pediatric audiology since the discovery of the Auditory Brainstem Response (ABR) in $1971^{1}$. Most prominently, these scalp recorded potentials have been utilized to screen for hearing loss and to estimate hearing thresholds in infants and young children who are unable to provide reliable responses with behavioral audiometry.

The number of pediatric patients requiring these tests has increased significantly since the widespread implementation of Universal Newborn Hearing Screening (UNHS) programs. Automated ABR screening devices are used globally in many UNHS programs to identify newborns that may be at-risk for hearing loss. Pediatric audiologists may now routinely see patients within the first few weeks of life for a diagnostic assessment of their hearing. The ABR has become the gold standard for 
diagnostic purposes to determine hearing thresholds in these infants ${ }^{2}$. This diagnostic information is the foundation of accountable intervention. It allows appropriate medical referral and informed decisions regarding amplification options (e.g. hearing aid, cochlear implant) and their subsequent programming and utilization, to be made. Ultimately, it serves to ensure optimal developmental outcomes for children diagnosed with hearing loss.

A more recent clinical AEP at the disposal of audiologists is the Auditory SteadyState Response (ASSR). Although first described three decades ago using slightly different methods, it only became available as a clinical tool for pediatric populations less than 10 years ago ${ }^{3}$. It was referred to by many different names including, the $40 \mathrm{~Hz}$ response, amplitude following response and steady stated evoked potential ${ }^{3}$. Its clinical value for pediatric populations before this time was very limited since responses were absent when subjects were asleep. Only once higher modulation frequencies were utilized, during the mid 90's, could children be tested during sleep. Since this discovery the ASSR has been investigated with great interest for its unique application possibilities. Current recommendations for diagnosis of hearing loss in infants have indicated that the ASSR should be used in combination with frequency-specific ABR measurements and not in place of these yet ${ }^{2}$. New evidence is however continually emerging in the validation of the ASSR as a reliable and accurate tool in diagnosis of hearing loss in infants. 


\section{BACKGROUND ON THE ASSR}

The ASSR is characterized by its periodic nature which is directly related to the periodic nature of the stimulus used to evoke the response. Figure 1 provides an illustration of this stimulus-response relationship. The most commonly used stimulus is a tone modulated in amplitude at a specific modulation frequency. The tone denotes the region of the basilar membrane being stimulated and is referred to as the carrier frequency or test frequency. The modulation frequency determines the rate of stimulation at the basilar membrane and is also the signature frequency used to identify if the response is present or not. In a sense the ASSR is a demonstration of how well the brain "follows" the periodic changes (modulation frequency) of the stimulus or conversely, how the stimulus "drives" the brain at a particular rate (modulation frequency) ${ }^{4}$. The rate of neural firing is therefore representative of the rate of stimulation. If a response is present, a peak of neural activity corresponding to the modulation frequency will be observed. Because the responses occur very rapidly as a function of the rapid stimulation, the brain's response to each stimulus is evoked before the response to the prior stimulus has ended. As a result the response does not return to a baseline state but remains a "steady-state" or sustained response.

\section{INSERT FIGURE 1}

The exact neural generators of the ASSR are not easy to identify since the whole nervous system is activated by modulated tones. Different regions of the auditory nervous system are however more involved depending on the rate of modulation ${ }^{4}$. 
As a general rule the cortex is the dominant source for rates below $20 \mathrm{~Hz}$ and the brainstem becomes increasingly predominant with rates greater than $50 \mathrm{~Hz}{ }^{4}$. The 40 $\mathrm{Hz}$ response generators have been attributed to the primary auditory cortices and thalamocortical circuits ${ }^{4}$.

\section{Stimuli}

The most commonly used stimulus for determining hearing thresholds is an amplitude modulated tone (Figure 1). Frequency modulation is commonly also added to increase the area of stimulation on the basilar membrane in an effort to increase the response amplitude. Other optional stimulus enhancements, such as exponential modulation, may also be available with the goal of increasing response amplitude to improve response detection.

An important advantage of the stimuli used in recording the ASSR above the ABR is the ability to simultaneously present multiple stimuli to both ears. As long as each stimulus is amplitude modulated at a different frequency. This multiple stimulus setup typically presents $500,1000,2000$ and $4000 \mathrm{~Hz}$ tones modulated at distinctly different rates to each ear simultaneously. The signature modulation frequency denotes the carrier frequency being assessed and therefore allows for simultaneous evaluation of up to eight frequencies (four in each ear). Multiple frequency stimulation is only recommended up to an intensity of approximately $80 \mathrm{~dB} H \mathrm{HL}$ where after interactions on the basilar membrane may reduce frequency-specificity and response amplitude. Typical stimulus parameters are provided in table 1.

\section{INSERT TABLE 1}




\section{Recording}

Current clinical ASSR systems typically use a single recording channel utilizing three scalp electrodes. The typical recording parameters and considerations for infants and children are provided in table 2 . The ASSR is a far-field potential and extremely small compared to the background electroencephalographic (EEG) noise. Improving the signal-to-noise ratio is achieved by adaptations to the stimulus (e.g. frequency modulation, exponential modulation etc), increasing the amount of averaging, including an artifact rejection criteria and making patient adaptations to ensure they are quiet and comfortable ${ }^{3}$. In young infants response amplitudes are largest when recorded from the ipsilateral mastoid. As a result, ff a single-channel AEP system is used it is best to record multiple frequencies simultaneously in one ear at a time ${ }^{3}$.

\section{INSERT TABLE 2}

The averaging approaches between clinical systems may differ. Some systems use a fixed averaging period (e.g. 90 seconds) for presentation of a stimulus independent of the stimulation intensity. Others use an adaptive averaging period that increase the averaging time as the intensity is decreased to compensate for the poorer signalto-noise ratio at lower intensities. The fixed averaging method requires an alternative way of compensating for elevated electrophysiological thresholds at lower intensities and employs a predictive formula to estimate hearing thresholds from the ASSR data 3. 
Recent reports have proposed the use of a fixed signal-to-noise ratio as a stop criterion as opposed to fixed time periods ${ }^{5,6}$. The noise levels recommended as a stop criterion varies from $5 \mathrm{nV}$ to $15 \mathrm{nV}^{3}$. Some noisy patients may not reach these levels within a clinically acceptable time frame and for such cases a maximum time limit should also be employed.

\section{Analysis}

Analysis of the ASSR is quite different to that of the ABR. In contrast to the ABR typically analyzed in the time-domain, the ASSR, is analyzed in the frequency domain. Utilizing higher modulation rates $(70-110 \mathrm{~Hz})$, necessary for reliable ASSR recordings in infants and young children, small amplitude responses are elicited that are difficult to recognize in the time-domain. Using a fast Fourier Transform (FFT) algorithm the amplitude and latency information in the time-domain is converted to the frequency-domain where recorded neural activity is represented as spectral components visualized in frequency and amplitude (see Figure 1).

Determining the presence of a response is also quite different for the ASSR compared to the ABR. Objective statistical methods are employed to determine whether a response was present in ASSR analysis as opposed to the subjective clinician analysis of an ABR recording. Two main approaches are used in objective response detection. The first evaluates the amplitude of the response in the frequency domain at the rate of modulation in relation to the background EEG in the adjacent regions (i.e. F-test). If a response is present it shows up as statistically significant at a level of $p<0.03$ or $p<0.05$. The second statistical procedure utilized by some systems uses the phase information contained at the response and is usually 
visualized on a vector plot. A response is present when the phases of each neural recording at the rate of modulation group together whilst it is absent if these phases are randomly distributed. A phase statistic (e.g. phase coherence) is used to determine if there is a significant difference (e.g. p<0.05) between the recorded phases and a typical random distribution.

\section{CLINICAL APPLICATION IN INFANTS \& CHILDREN}

\section{Screening}

The ASSR's objective detection methods and the possibility of simultaneously testing multiple frequencies in both ears make it an appealing hearing screening technology. Current methods such as automated $A B R$ and oto-acoustic emission (OAE) screening are however, very well established with excellent sensitivity and specificity. Any ASSR screening technology will therefore have to clearly demonstrate at least the same level of reliability and efficiency. New systems are being developed that employ the ASSR automated response detection methods to the presentation of broadband stimuli such as clicks, chirps and white noise. The use of low and high frequency stimuli (e.g. filtered noise or chirps) simultaneously presented to screen for hearing loss may be very useful to differentiate referrals due to middle-ear effusion and also to identify low frequency hearing loss often missed by a click ABR. At present however ASSR-based screening methods will have to show comparative results or additional advantages compared to the gold standard of automated $\mathrm{ABR}^{3}$. 


\section{Air conduction thresholds}

Hearing thresholds have effectively been estimated by ASSR recordings in infants and young children. A maturation effect is observed for ASSR in normal hearing infants compared to adults. Infant thresholds are typically elevated by between 10 to $15 \mathrm{~dB}$, most prominently at 500 followed by $4000 \mathrm{~Hz}^{7-13}$. Current evidence suggests the maturational effects are most prominent in the first few weeks of life dissipating within the first three months ${ }^{6,7,14}$. A recent study comparing ASSR thresholds for preterm and term infants revealed a significant difference between thresholds at 500 and $4000 \mathrm{~Hz}$ with slightly higher thresholds for preterm infants $\left({ }^{7}\right.$. The maturational effect has been attributed to a combination of neural immaturity, vernix in the ear canal and developmental changes in the acoustics of the ear canal or middle-ear ${ }^{8,12,15}$. In a more recent study no significant differences in ASSR thresholds were noted in normal hearing infants younger than 6 months and those older than 6 months ${ }^{6}$. Comparing ASSR thresholds between a sub-group of the 10 youngest infants ( 1 to 3 months of age) and the other infants revealed no significant differences in this study. Current evidence therefore suggest that the ASSR may reliably confirm normal hearing in infants from 4 to 12 weeks of age ${ }^{6,12,16}$. The expected normal hearing levels for ASSR thresholds in infants beyond the first few weeks of life is $50,45,40$ and $40 \mathrm{~dB}$ at $500,1000,2000$ and $4000 \mathrm{~Hz}^{6}$.

Research validation for ASSR thresholds in children with hearing loss, especially with the multiple stimulus technique, has been insufficient to date. Existing data suggest that ASSR thresholds can be obtained significantly closer to behavioral thresholds with increasing sensorineural hearing loss severity. This has been attributed to a physiological recruitment phenomenon that results in higher amplitude 
responses close to threshold in cases of hearing loss compared to normal hearing subjects $^{8,17}$. The reliability of air conduction ASSR thresholds for conductive hearing loss in infants is not clear but adult studies indicate that thresholds are reliable and representative ${ }^{5}$. Differentiating between mild sensorineural hearing loss and normal hearing and mild and moderate sensorineural hearing loss in adults suggest that this may be difficult especially at 500 and $2000 \mathrm{~Hz}^{5}$.

A single-stimulus ASSR study in newborns, comparing 500 and $2000 \mathrm{~Hz}$ tone-burst ABR and ASSR thresholds, indicated that ASSR thresholds are elevated and more variable in the first 6 weeks of life ${ }^{18}$. The reported differences between the two techniques seem to disappear by 6 weeks of age. A recent study did similar comparisons between the multiple ASSR technique and tone burst ABR thresholds in infants between 2 and 36 months of age with sensorineural hearing loss ${ }^{19}$. The tone burst ABR and ASSR thresholds at 500,1000, 2000 and $4000 \mathrm{~Hz}$ were highly correlated with mean differences of between -0.2 and $-2.8 \mathrm{~dB}$ and standard deviations between 3.9 and $6.4 \mathrm{~dB}^{19}$. The tone burst ABR and ASSR thresholds were also compared to behavioral thresholds that were recorded at a later stage. Although the sample size was small, results indicate similar correlations between the electrophysiological thresholds of the two techniques compared to behavioral thresholds ${ }^{19}$.

A possible advantage of the ASSR is the fact that the modulated tones may provide a better estimation of steeply sloping high-frequency hearing loss than frequencyspecific $A B R{ }^{3}$. The nature of the ASSR stimuli also allows for assessing hearing loss at intensities exceeding the maximum ABR output limit of approximately $95 \mathrm{~dB}$ 
$\mathrm{nHL}^{20}$. Assessing hearing at levels of up to $120 \mathrm{~dB} \mathrm{HL}$ make it possible to differentiate severe and profound hearing losses, which cannot be achieved with the ABR. This advantage was brought into question due to reports indicating spurious or artifactual ASSR thresholds at these high intensities in the absence of any behavioral thresholds ${ }^{21,22}$. These responses were not generated in the auditory system and were attributed primarily to the relatively slow analog-to-digital (A/D) rates $(500$ and $1000 \mathrm{~Hz}$ ) employed by early systems. This resulted in aliasing of the stimulus artifact to the recording if carrier frequencies were integers of the $A / D$ rate ${ }^{2221}$. Newer systems and software versions have now changed $A / D$ sampling rates and added steep anti-aliasing filters to avoid these artifactual responses. Using single-polarity air conducted stimuli with an A/D rate of $1250 \mathrm{~Hz}$ allow accurate threshold determination for all frequencies, except perhaps $1000 \mathrm{~Hz}$, up to at least 114 to $120 \mathrm{~dB} \mathrm{HL}^{23}$. Another possible source of spurious responses in the low frequencies $(<1000 \mathrm{~Hz})$ is the vestibular system. Responses at $500 \mathrm{~Hz}$ seem to be biologic in nature since they do not invert with reverse stimulus polarity. Highintensity thresholds (>95 dB HL) should be therefore be interpreted with caution at this frequency ${ }^{3,22}$.

\section{Bone conduction thresholds}

Including auditory evoked potentials to measure bone conduction thresholds provides a way of differentiating types of hearing loss and should be a part of routine diagnostic procedures for infants ${ }^{2}$. Current research indicates that reliable bone conduction thresholds can be obtained in preterm, term and older infants with normal hearing ${ }^{24-27}$. A maturational effect suggests that bone conduction ASSR thresholds improve in the high frequencies and deteriorate in the low frequencies as illustrated 
in figure 2. A series of studies investigating the procedural factors that may influence the measurement of bone conduction ASSR thresholds in infants ${ }^{24-27}$ recommend the following: (1) Coupling the bone oscillator can be done with an elastic band or handheld with training; (2) Forehead placement should be avoided but temporal and mastoid placement is appropriate; (3) Occlusion does not have a significant effect on threshold estimation; (4) Interaural attenuation for infants with bone conduction is greater than for adults (at least 10 to $30 \mathrm{~dB}$ ) making isolation of the test cochlea easier.

\section{INSERT FIGURE 2}

An important concern in bone conduction ASSR measurement is the presence of artifactual responses at reasonably low stimulation levels as illustrate in figure $2^{27,28}$. Artifactual responses are due to the same reasons as spurious air conduction thresholds at high intensities (discussed in previous section). Bone conduction ASSR thresholds recorded in a sample of infants with severe-to-profound sensorineural hearing loss indicate that these response may begin to occur at 25, 40, 60, 60 and $60 \mathrm{~dB}$ for $250,500,1000,2000$ and $4000 \mathrm{~Hz}$ respectively ${ }^{27}$. Although not confirmed in actual investigations in infants to date, there are procedural adaptations to the bone conduction test setup that may reduce the stimulus artifact. These include selecting more appropriate A/D conversion rates (e.g. $1250 \mathrm{~Hz}$ ), braided electrode wires, and using a grounded screen for the bone conduction transducer and its cable

29. Alternatively the Sensitivity Acuity Level (SAL) technique can be adapted for the ASSR to estimate bone conduction thresholds ${ }^{30}$. This technique may however be 
open to more variability since bone conduction thresholds are derived from two estimated thresholds.

Bone conduction ASSR thresholds in infants may be useful to categorize normal sensorineural hearing in infants but the occurrence of artifactual responses at low levels make differentiating types and degrees of hearing loss difficult. Current evidence suggest that sensorineural hearing loss of a mild or greater degree in the low frequencies $(\leq 500 \mathrm{~Hz})$ and of a moderate and greater degree in the high frequencies $(\geq 1000 \mathrm{~Hz})$, cannot be quantified confidently ${ }^{27}$. More research data is necessary to validate and compare bone conduction ASSR testing in infants and children compared to frequency-specific ABR before it can be recommended for widespread clinical use.

\section{Sound-field thresholds}

The continuous modulated tones, used to evoke the ASSR, are less likely to distort when presented through sound-field speakers or when processed by a hearing aid than transient stimuli used for ABR recordings. Successful recordings of aided ASSR thresholds that correlate reasonably well with behavioral thresholds have been reported for adults and infants ${ }^{31,32}$. Aided ASSR thresholds recorded in a group of infants demonstrated the largest variability at $500 \mathrm{~Hz}$ with more than half of these thresholds being absent during sound-field recordings ${ }^{31}$. Other frequencies were consistently present and this information provided the first robust evidence of hearing aid benefit in these young infants who were unable to provide reliable behavioral responses at that time ${ }^{31}$. This type of information may be very valuable to guide 
case management, especially in light of the increasingly younger population being served by audiologists with the widespread implementation of UNHS.

\section{Auditory Neuropathy}

This auditory condition reportedly constitutes approximately $10 \%$ of all permanent hearing loss cases ${ }^{33,34}$. It is characterized by evidence of cochlear functioning by the presence of a cochlear microphonic response and/or present oto-acoustic emissions. The ABR in contrast is totally absent or present at high intensities but grossly abnormal. These findings typically result in varying degrees of hearing loss and perceptual difficulties related to temporal processing. ASSR thresholds in cases of auditory neuropathy do not demonstrate correlation to behavioral thresholds and if

present are typically elevated by 30 to $40 \mathrm{~dB}^{17,35,35-38}$. As a result, ASSR thresholds in cases of auditory neuropathy do not predict hearing thresholds and neither can the ASSR diagnose the condition. The ABR remains essential in diagnosing the presence of auditory neuropathy although, as with the ASSR, it cannot predict hearing thresholds for these patients.

\section{CONCLUSION}

The ASSR has been used clinically for close to a decade and has proved itself to be a valuable part of the audiological test-battery in pediatric audiology. The longstanding research and clinical validation history of the frequency-specific ABR make it the current gold standard for estimating hearing thresholds in young infants and children ${ }^{2}$ but accumulating evidence is establishing the ASSR in its own right. 
ASSR may offer unique advantages such as its frequency-specific determination of auditory functioning with the capability of multiple frequencies simultaneously assessed in both ears without the need for a clinician to interpret responses. The continuous nature of ASSR stimuli allow for higher intensities (exceeding $100 \mathrm{~dB}$ $H L)$, where ABR thresholds may be absent, to be assessed and for aided thresholds to be recorded. Although more evidence is necessary, reports are indicating close correlations between air conduction ABR and ASSR thresholds ${ }^{39}$. More validation data is however required in infants and children with hearing loss whilst bone conduction testing should be optimized to reduce artifactual responses and increase the effective range of stimulation.

At present the ASSR is an invaluable adjunct to the ABR. It may be utilized to improve the efficiency of test-protocols, supplementing ABR data and cross-checking test results. The efficiency the audiological test-battery may be improved by employing the ASSR to determine if infants have responses at normal hearing levels across several frequencies assed simultaneously in both ears ${ }^{3}$. If responses are present frequency-specific ABR measures may be omitted from the test-battery. It can supplement the test-battery when ABR thresholds are absent at maximum intensities since the ASSR can stimulate at higher intensities. This information could differentiate severe and profound hearing losses and direct amplification and intervention decisions more precisely.

\section{REFERENCES}

1. Jewett DL, Williston JS. Auditory-evoked far fields averaged from the scalp of humans. Brain. 1971;94(4):681-696. 
2. Busa J, Harrison J, Chappell J, et al. Year 2007 position statement: Principles and guidelines for early hearing detection and intervention programs. Pediatrics. 2007;120(4):898-921.

3. Hall JW, III, Swanepoel D. Objective assessment of hearing. San Diego: Plural Publishing; 2010:186.

4. Picton TW, John MS, Dimitrijevic A, Purcell D. Human auditory steady-state responses. International Journal of Audiology. 2003;42(4):177-219.

5. D'haenens W, Vinck BM, Maes L, et al. Determination and evaluation of clinically efficient stopping criteria for the multiple auditory steady-state response technique. Clinical Neurophysiology. 2010.

6. Van Maanen A, Stapells DR. Normal multiple auditory steady-state response thresholds to air-conducted stimuli in infants. J Am Acad Audiol. 2009;20(3):196-207.

7. Ribeiro FM, Carvallo RM, Marcoux AM. Auditory steady-state evoked responses for preterm and term neonates. Audiology and Neurotology. 2010;15(2):97-110.

8. Luts H, Desloovere C, Wouters J. Clinical Application of Dichotic Multiple-Stimulus Auditory Steady-State Responses in High-Risk Newborns and Young Children. Audiol Neurootol. 2006;11(1):24-37.

9. Rance G, Rickards F. Prediction of Hearing Threshold in Infants Using Auditory Steady-State Evoked Potentials. J Am Acad Audiol. 2002;13(5):236.

10. Cone-Wesson B, Parker J, Swiderski N, Rickards F. The Auditory Steady-State Response: Full-Term and Premature Neonates. J Am Acad Audiol. 2002;13(5):260. 
11. Swanepoel W, Steyn K. Short report: establishing normal hearing for infants with the auditory steady-state response. The South African journal of communication disorders.Die Suid-Afrikaanse tydskrif vir Kommunikasieafwykings. 2005;52:36-39.

12. John MS, Brown DK, Muir PJ, Picton TW. Recording auditory steady-state responses in young infants. Ear Hear. 2004;25(6):539-553.

13. Lins OG, Picton TW, Boucher BL, et al. Frequency-specific audiometry using steady-state responses. Ear Hear. 1996;17(2):81-96.

14. Alaerts J, Luts H, Van Dun B, Desloovere C, Wouters J. Latencies of auditory steady-state responses recorded in early infancy. Audiology and Neurotology. 2010;15(2):116-127.

15. Rance G, Tomlin D. Maturation of auditory steady-state responses in normal babies. Ear Hear. 2006;27(1):20-29.

16. Rance G, Tomlin D. Maturation of auditory steady-state responses in normal babies. Ear Hear. 2006;27(1):20-29.

17. Rance G, Roper R, Symons L, et al. Hearing Threshold Estimation in Infants Using Auditory Steady-State Responses. J Am Acad Audiol. 2005;16(5):291-300.

18. Rance G, Tomlin D, Rickards FW. Comparison of auditory steady-state responses and tone-burst auditory brainstem responses in normal babies. Ear Hear. $2006 ; 27(6): 751-762$. 
19. Rodrigues GRI, Lewis DR. Threshold prediction in children with sensorioneural hearing loss using the auditory steady-state responses and tone-evoked auditory brain stem response. Int J Pediatr Otorhinolaryngol. 2010;74(5):540-546.

20. Swanepoel D, Hugo R, Roode R. Auditory Steady-State Responses for Children with Severe to Profound Hearing Loss. Archives of Otolaryngology - Head and Neck Surgery. 2004;130(5):531-535.

21. Gorga MP, Neely ST, Hoover BM, Dierking DM, Beauchaine KL, Manning C. Determining the upper limits of stimulation for auditory steady-state response measurements. Ear Hear. 2004;25(3):302-307.

22. Small SA, Stapells DR. Artifactual responses when recording auditory steadystate responses. Ear Hear. 2004;25(6):611-623.

23. Picton TW, John MS. Avoiding electromagnetic artifacts when recording auditory steady-state responses. J Am Acad Audiol. 2004;15(8):541-554.

24. Small SA, Stapells DR. Multiple auditory steady-state response thresholds to bone-conduction stimuli in young infants with normal hearing. Ear Hear. 2006;27(3):219-228.

25. Small SA, Hatton JL, Stapells DR. Effects of bone oscillator coupling method, placement location, and occlusion on bone-conduction auditory steady-state responses in infants. Ear Hear. 2007;28(1):83-98.

26. Small SA, Stapells DR. Normal ipsilateral/contralateral asymmetries in infant multiple auditory steady-state responses to air- and bone-conduction stimuli. Ear Hear. 2008;29(2):185-198. 
27. Swanepoel DW, Ebrahim S, Friedland P, Swanepoel A, Pottas L. Auditory steady-state responses to bone conduction stimuli in children with hearing loss. Int J Pediatr Otorhinolaryngol. 2008;72(12):1861-1871.

28. Fuh-Cherng Jeng, Brown CJ, Johnson TA, Vander Werff KR. Estimating AirBone Gaps Using Auditory Steady-State Responses. J Am Acad Audiol. 2004;15(1):67-78.

29. Brooke RE, Brennan SK, Stevens JC. Bone conduction auditory steady state response: Investigations into reducing artifact. Ear Hear. 2009;30(1):23-30.

30. Cone-Wesson B, Rickards F, Poulis C, Parker J, Tan L, Pollard J. The Auditory Steady-State Response: Clinical Observations and Applications in Infants and Children. J Am Acad Audiol. 2002;13(5):270.

31. Stroebel D, Swanepoel D, Groenewald E. Aided auditory steady-state responses in infants. International Journal of Audiology. 2007;46(6):287-292.

32. Picton TW, Durieux-Smith A, Champagne SC, et al. Objective evaluation of aided thresholds using auditory steady-state responses. J Am Acad Audiol. 1998;9(5):315-331.

33. Rance G. Auditory neuropathy/dys-synchrony and its perceptual consequences. Trends in Amplification. 2005;9(1):1-43.

34. Sininger YS. Identification of auditory neuropathy in infants and children. Seminars in Hearing. 2002;23(3):193-200. 
35. Rance G, Briggs RJS. Assessment of Hearing in Infants with Moderate to Profound Impairment: the Melbourne Experience with Auditory Steady-State Evoked Potential Testing. Annals of Otology, Rhinology \& Laryngology. 2002;111:22.

36. Rance G, Beer DE, Cone-Wesson B, et al. Clinical findings for a group of infants and young children with auditory neuropathy. Ear Hear. 1999;20(3):238-252.

37. Emara AA, Gabr TA. Auditory steady state response in auditory neuropathy. J Laryngol Otol. 2010:1-7.

38. Jafari Z, Malayeri S, Ashayeri H, Farahani MA. Adults with auditory neuropathy: Comparison of auditory steady-state response and pure-tone audiometry. J Am Acad Audiol. 2009;20(10):621-628.

39. Rodrigues GRI, Lewis DR. Threshold prediction in children with sensorioneural hearing loss using the auditory steady-state responses and tone-evoked auditory brain stem response. Int J Pediatr Otorhinolaryngol. 2010. 
Table 1. Typical ASSR stimulus parameters for infants and children

\begin{tabular}{|c|c|}
\hline Parameter & Selection \\
\hline Carrier frequencies & $500,1000,2000,4000 \mathrm{~Hz}$ \\
\hline \multirow[t]{3}{*}{ Modulation frequencies } & $70-100 \mathrm{~Hz}$ \\
\hline & Multiple simultaneous stimuli carrier tones \\
\hline & modulated at distinct rates \\
\hline Amplitude modulation (AM) depth & $100 \%$ \\
\hline Frequency modulation (FM) depth & $10-20 \%$ \\
\hline Advanced modulation options & Exponential modulation $\left(\mathrm{AM}^{2}\right)$ \\
\hline \multirow[t]{4}{*}{ Stimulus intensity range } & 0-125 dB HL (depending on transducer and \\
\hline & frequency) \\
\hline & Simultaneous multiple stimuli $\leq 80 \mathrm{~dB} \mathrm{HL}$ \\
\hline & Single stimuli $>80 \mathrm{~dB} H \mathrm{HL}$ \\
\hline \multirow[t]{2}{*}{ Transducers } & Insert earphones, supra-aural earphones, sound- \\
\hline & field speaker, bone oscillator \\
\hline Calibration reference & $\mathrm{dB} H \mathrm{HL}$ \\
\hline
\end{tabular}


Table 2. Typical ASSR recording parameters for infants and children

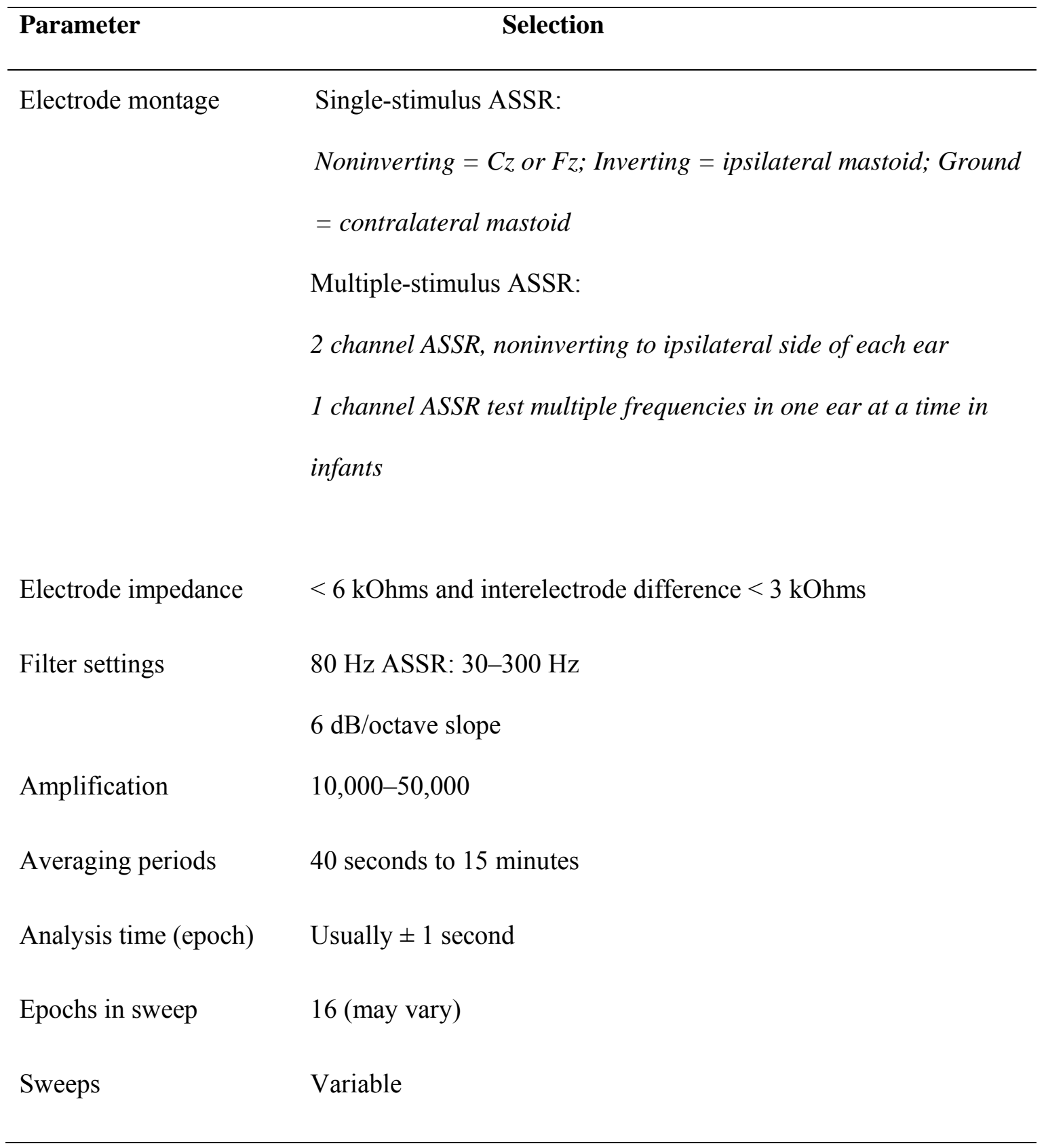




\section{Stimulus $\longrightarrow$ Cochlea $\longrightarrow \begin{gathered}\text { EEG time } \\ \text { domain }\end{gathered} \longrightarrow \begin{gathered}\text { EEG frequency } \\ \text { domain }\end{gathered}$}

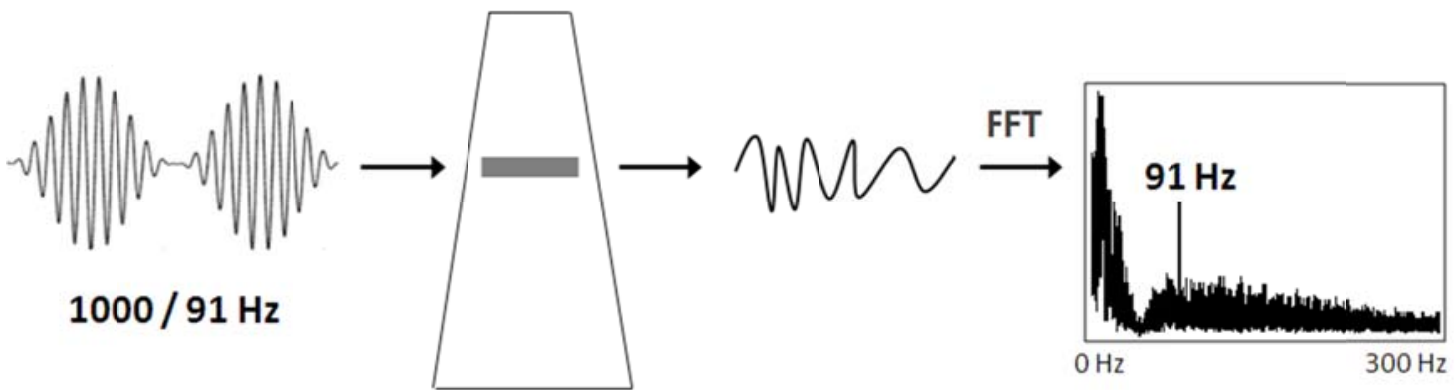

Figure 1. ASSR stimulus-response relationship. The $1000 \mathrm{~Hz}$ carrier tone is amplitude modulated at $91 \mathrm{~Hz}$. The carrier tone $(1000 \mathrm{~Hz})$ determines the region of the basilar membrane being stimulated and the modulation rate designates the rate of stimulation. Recorded EEG activity represented in the time-domain is converted to the frequency-domain using a fast Fourier Transform (FFT). The recorded neural activity is plotted as a function of amplitude and frequency (phase information is also preserved) and indicates a response peak at the rate of stimulation $(91 \mathrm{~Hz})$. 


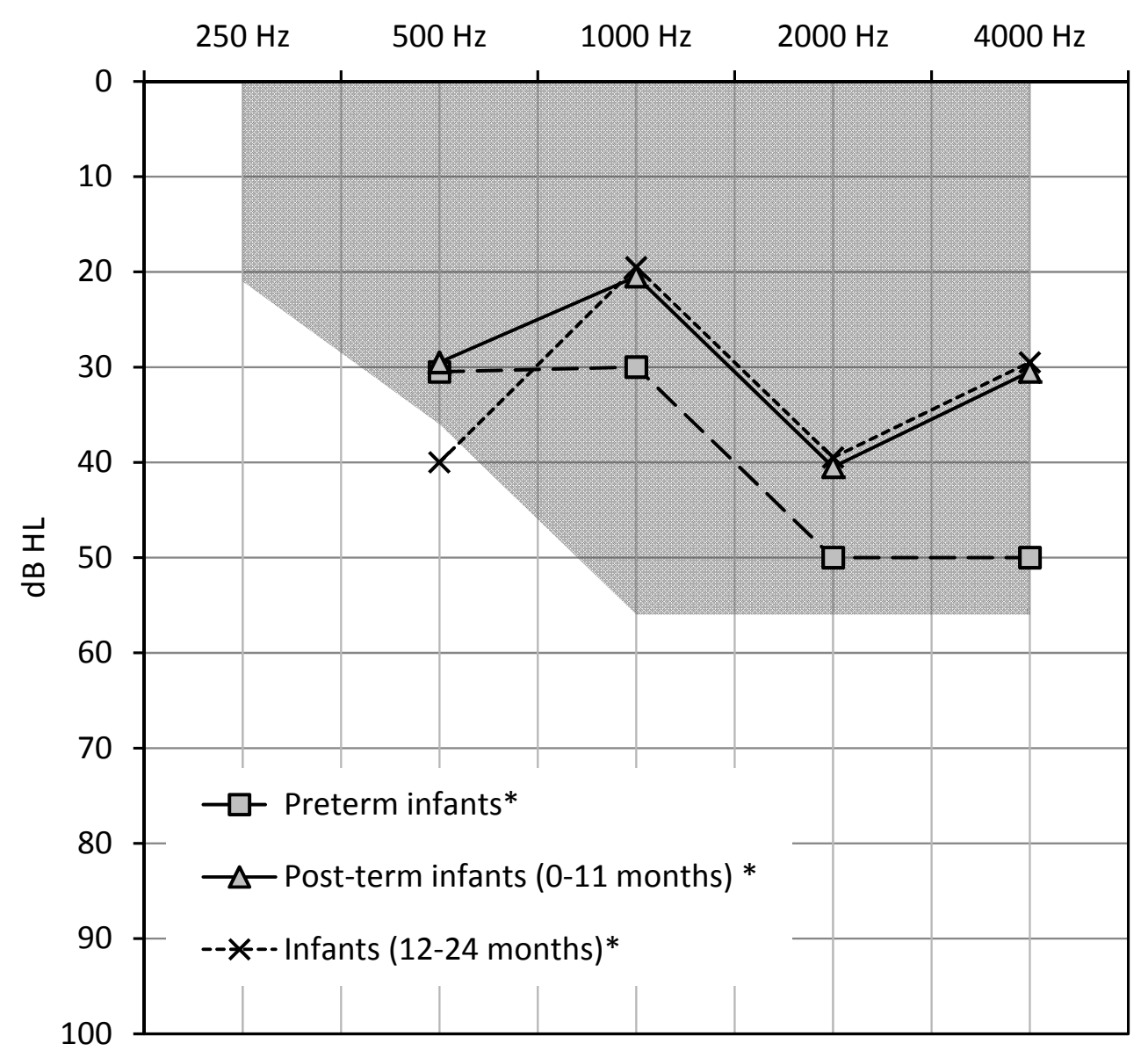

Figure 2. Effective range for bone conduction testing and normal threshold levels for infants. Shaded area denotes the range in which artifactual ASSR thresholds do not occur (Source: Swanepoel et al. ${ }^{27}$ ). *More than $90 \%$ of infants with normal hearing had thresholds at theses levels. (Source: Small \& Stapells ${ }^{24,26}$ ) 